\title{
HAADF and EELS Combined Studies of a New Generation of Materials for Ni- MH Batteries
}

\author{
V. Serin ${ }^{1,2}$, C. Magen ${ }^{2,3}$, R. Serra ${ }^{1}$, M. R. Ibarra ${ }^{2,3}$, M.J. Hÿtch ${ }^{1,2}$, J. Zhang ${ }^{4}$, L. Lemort ${ }^{4}$, M. \\ Latroche $^{4}$, B. Knosp 5 , and P. Bernard ${ }^{5}$ \\ 1 CEMES-CNRS, TALEM, 29 rue Jeanne Marvig, 31055 Toulouse, France \\ 2 TALEM, CNRS-Universidad de Zaragoza, 29 rue Jeanne Marvig, 31055 Toulouse, France \\ 3 Instituto de Nanociencia de Aragón, Departamento de Física de la Materia Condensada, \\ Universidad de Zaragoza, 50009, Zaragoza, Spain \\ 4 CMTR, ICMPE-CNRS, 2-8 rue Henri Dunant 94320 Thiais, France \\ 5 SAFT, Direction de la Recherche, 111-113 bld. Alfred Daney, 33074 Bordeaux France \\ corresponding author: serin@cemes.fr
}

Applications for Ni-MH batteries are driven by increased energy density. Hydride forming compounds such as $\mathrm{La}-\mathrm{Mg}-\mathrm{Ni}$ ternary compounds show improved performance, in particular new crystallographic phases of $\mathrm{A}_{2} \mathrm{~B}_{7}$ type $[1,2,3]$. Such compounds can be described as the growth along the $\mathrm{c}$ axis of two different sub-units $\left[\mathrm{AB}_{5}\right]$ ( $C$ layer) and $\left[\mathrm{A}_{2} \mathrm{~B}_{4}\right]$ ( $L$ layer), where $\mathrm{A}$ is a rare earth or an alkali earth and $\mathrm{B}$ is a transition metal, forming either a rhombohedral $[3 R](R-3 m)$ or hexagonal $[2 \mathrm{H}]\left(\mathrm{P}_{3} / \mathrm{mmc}\right)$ crystallographic structure. A series of compounds in this family of materials has been prepared by spark plasma sintering (SPS) and characterized by X-ray, microprobe and TEM analysis.

Here we present analysis of a $\mathrm{La}_{0.65} \mathrm{Nd}_{0.15} \mathrm{Mg}_{0.20} \mathrm{Ni}_{3.5}$ compound by HREM, HAADF and STEM EELS in order to identify the local structure and defects. HRTEM observations were performed on an image-corrected FEI Tecnai (SACTEM-Toulouse) operating at $200 \mathrm{kV}$ and HAADF imaging and STEM EELS on a probe-corrected FEI Titan 60-300 microscope operating at $300 \mathrm{kV}$ (Zaragoza).

Assuming the intensity of the columns can be related to the $Z$ atomic numbers of the different elements in presence (Nd:60, La:57, Ni:28, Mg:12), the structure can be interpreted readily from the HAADF image from Fig. 1. In particular, the highest intensity can be identified as the rare-earth columns, and the layers lacking contrast the nominally $\mathrm{Mg}_{2} \mathrm{Ni}_{4}$ layers. The fact that the $\mathrm{Mg}_{2} \mathrm{Ni}_{4}$ layer are visible at all, suggests that rare-earth elements are indeed also present here. This is confirmed by $\mathrm{X}$-ray analysis showing that $\mathrm{Mg}$ partially substitutes rare-earth elements only in the $\left[\mathrm{A}_{2} \mathrm{~B}_{4}\right]$ layer. The contrast allows us to identify the structure in this grain as the rhomohedral $[3 R]$ structure in agreement with the majority phase determined from Rietveld analysis of the X-ray diffraction patterns. As expected, the Ni sublattice is less identifiable, though the columns with two superposed $\mathrm{Ni}$ atoms in the unit cell are clearly visible. The intensity profile along the Ni atom line (in red on Fig 1), which is superimposed to the HAADF image, is of help to discriminate single and superposed $\mathrm{Ni}$ atoms. The simulation fits quite well the image, but the exact position of $\mathrm{La}$ and $\mathrm{Nd}$ atoms remains unclear (close $Z$ values). Spatially resolved STEM EELS cartography will be presented to conclude on that point and on the chemical nature of occasional changes in the sequence of stacking. The spatially resolved EELS analysis will be helpful to accurately interpret the contrast in the HAADF images.

References

[1] T. Kohno et al., Journal of Alloys and Compounds 311 (2000) L5.

[2] E. Akiba, H. Hayakawa, and T. Kohno, Journal of Alloys and Compounds 408-412 (2006) 280.

[3] R. V. Denys et al., Journal of Solid State Chemistry 181 (2008) 812.

[4] This work is supported by the ANR program MAHYA ( ${ }^{\circ}$ ANR-07-STOCK-E-MAHYA-07-01). 

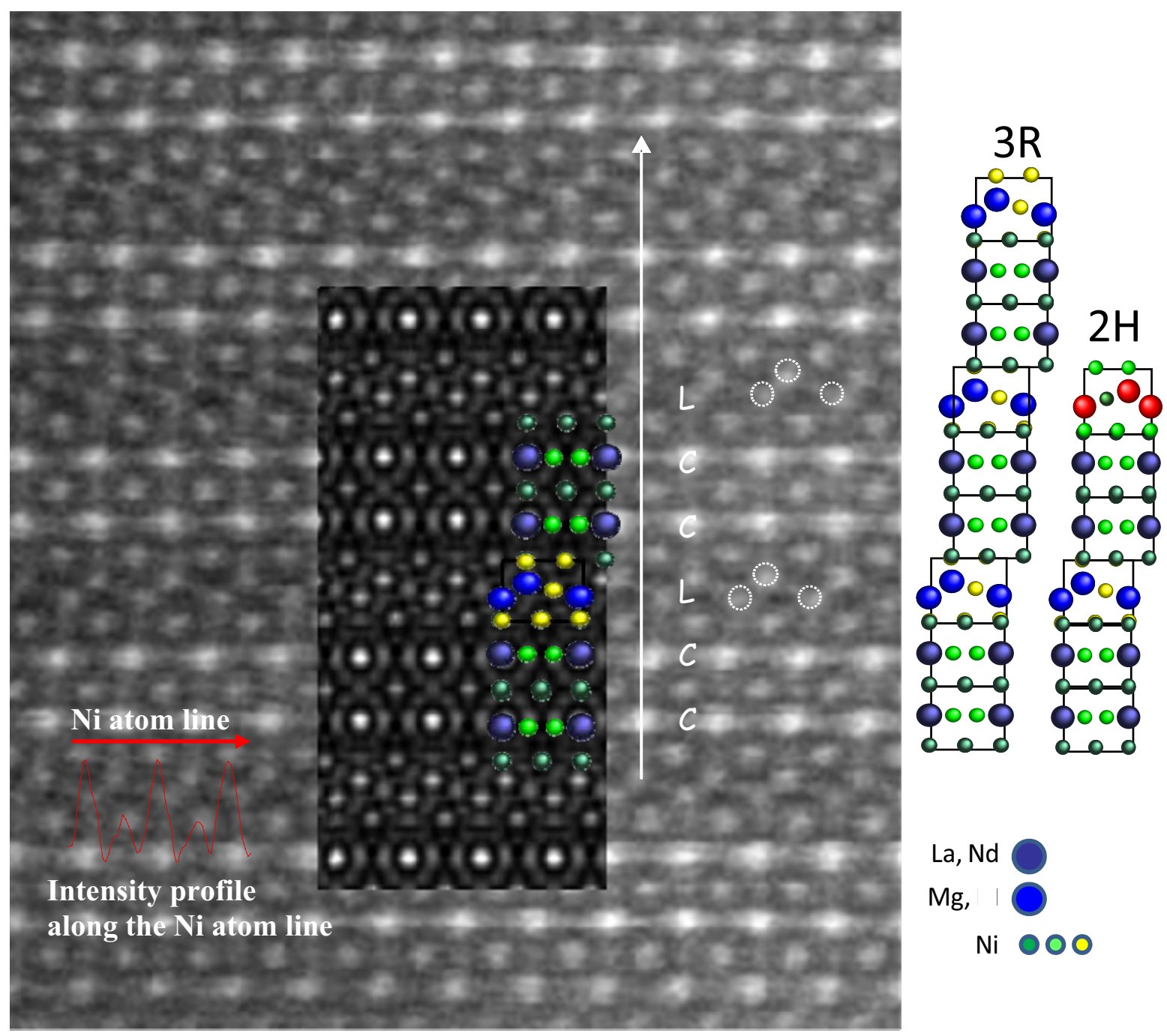

FIG. 1. : Ultra high magnification HAADF image of the $\mathrm{La}_{0.65} \mathrm{Nd}_{0.15} \mathrm{Mg}_{0.20} \mathrm{Ni}_{3.5}$ sample. A simulation and the structure are superimposed. The intensity variation along a Ni line (indicated by a red arrow) is also superimposed. The image has been Wiener filtered. 